\title{
PROJECTION-BASED REDUCED ORDER MODEL FOR PRESTRESSED HYDROELASTIC VIBRATIONS
}

\author{
C. HOAREAU, J.-F. DEÜ AND R. OHAYON \\ Laboratoire de Mécanique des Structures et des Systèmes Couplés (LMSSC) \\ Conservatoire national des arts et métiers \\ 292 Rue Saint-Martin, 75141 Paris, France \\ e-mail: christophe.hoareau@lecnam.net, web page: http://www.lmssc.cnam.fr/en
}

Key words: Fluid-Structure interaction, Reduced Order Model, Geometric Nonlinearity

\begin{abstract}
In aerospace industry, the computations of liquid-propelled launchers vibrations are based on linear elastic tanks coupled with an inviscid, incompressible fluid with free surface. In this specific case, the fluctuation of pressure on the free surface is supposed to be zero (i.e. the sloshing effects are neglected). Those vibrations are usually called hydroelastic vibrations. The discretized finite element dynamic problem is commonly expressed only in terms of displacements considering an added mass matrix, which represents the kinetic energy of the moving liquid expressed in terms of normal displacement interface. Those computations can be performed for various fluid level configurations, but no prestressing from geometrical nonlinearity is usually considered [1]. In the present study, the evaluation of the prestressed state influence on the coupled fluid structure vibrations is estimated numerically. This prestressing is supposed to be due to a gas or liquid pressurization, acting on the internal surface of the tank, inducing relatively large static displacement compared to the tank thickness. In reference [2], we have evaluated efficiently and accurately the nonlinear displacements for various filling rate with the use of an original level-set approach. We present here the hydroelastic vibrations around each known prestressed state corresponding to different level of liquid with the use of the added mass matrix. Using the open-source computing finite element platform FEniCS [3], numerical results are in very good agreements with experimental studies from the literature [4]. Comparisons with and without prestressing illustrates the contribution of the effect. To overcome an expensive added mass matrix computation, an appropriate reduced order model obtained by projection on prestressed dry modes is also proposed and show very encouraging results.
\end{abstract}




\section{INTRODUCTION}

The subject consists in computing the fluid-structure dynamic behavior of prestressed elastic tanks containing a free surface liquid. An original expression of a reduced eigenvalue problem involving: (i) a reduced tangent matrix and (ii) a reduced added mass matrix, allow a fast computation of the eigenfrequencies for each level of liquid height. The problem is presented in two steps (see Fig. 5). First, nonlinear static problems with geometrical nonlinearities and follower forces are solved with the finite-element (FE) method. Then a linearized dynamic problem is proposed and solved considering the projection-based reduced order model.

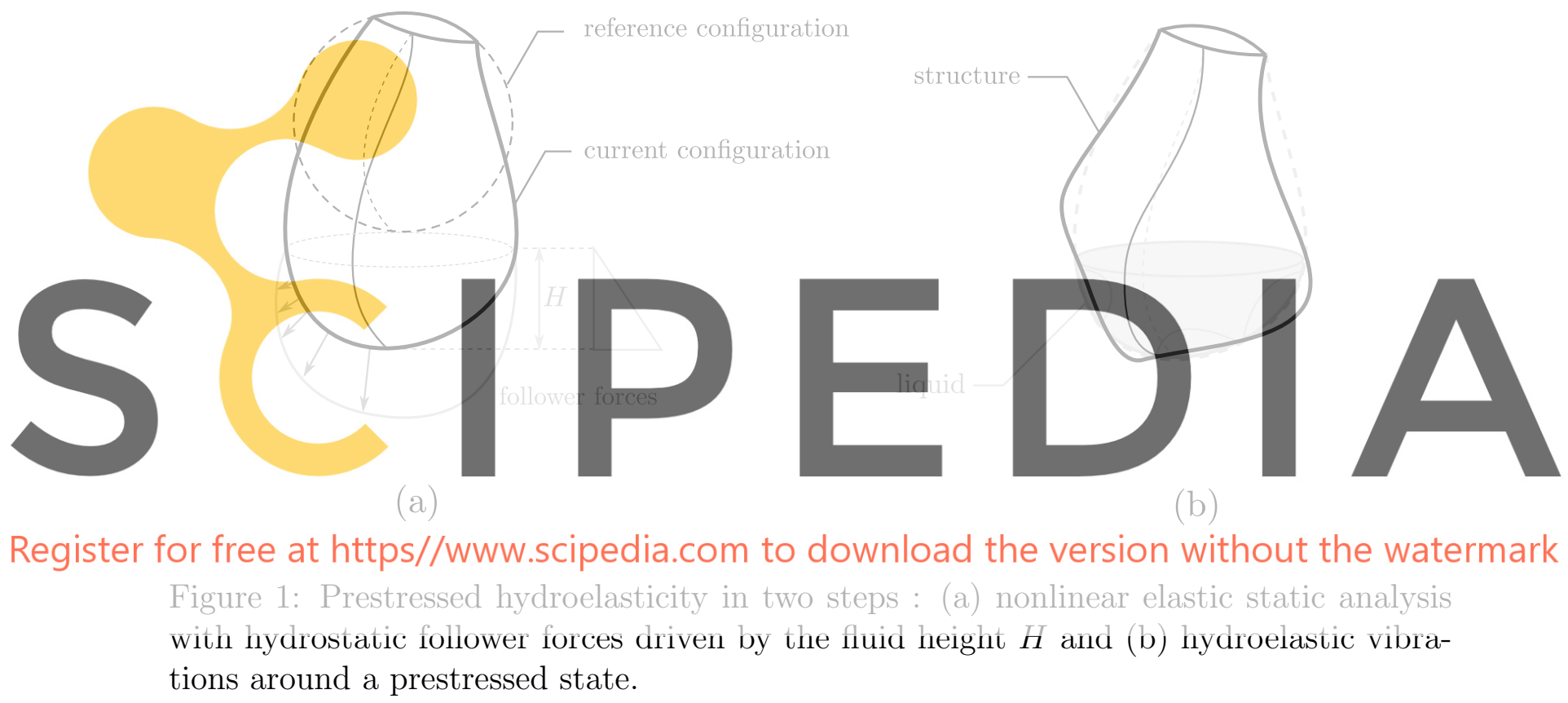

\section{Nonlinear static problem with hydrostatic follower forces}

\subsection{Assumptions in the solid domain}

The structural reference domain, denoted as $\Omega_{\mathrm{s}}$, is supposed to be homogeneous and isotropic. Considering a mechanical solicitation (i.e. a hydrostatic follower forces in this case), the problem consists in finding the resulting structural displacement field $\boldsymbol{u}_{\mathrm{s}}$ at equilibrium considering a given fluid height. The index "s" is a notation attributed to the static displacement solution of a nonlinear problem with geometric nonlinearities. 


\subsection{Preliminary definitions of nonlinear elasticity}

We recall the definition of the Green-Lagrange strain tensor and its virtual form:

$$
\begin{aligned}
\boldsymbol{E}\left(\boldsymbol{u}_{\mathrm{s}}\right) & =\frac{1}{2}\left(\operatorname{Grad}^{\mathrm{T}} \boldsymbol{u}_{\mathrm{s}}+\operatorname{Grad} \boldsymbol{u}_{\mathrm{s}}+\operatorname{Grad}^{\mathrm{T}} \boldsymbol{u}_{\mathrm{s}} \operatorname{Grad} \boldsymbol{u}_{\mathrm{s}}\right) \\
\delta \boldsymbol{E}\left(\boldsymbol{u}_{\mathrm{s}}, \delta \boldsymbol{u}\right) & =\frac{1}{2}\left(\operatorname{Grad}^{\mathrm{T}} \delta \boldsymbol{u}+\operatorname{Grad} \delta \boldsymbol{u}+\operatorname{Grad}^{\mathrm{T}} \delta \boldsymbol{u} \operatorname{Grad} \boldsymbol{u}_{\mathrm{s}}+\operatorname{Grad}^{\mathrm{T}} \boldsymbol{u}_{\mathrm{s}} \operatorname{Grad} \delta \boldsymbol{u}\right)
\end{aligned}
$$

where the gradient operator Grad is given in the reference configuration of the structure $\Omega_{\mathrm{s}}$ and $\delta \boldsymbol{u}$ is a virtual displacement function. In the following, the second Piola-Kirchhoff stress tensor $\boldsymbol{S}$ is supposed to be obtained considering a linear Saint-Venant Kirchhoff (SVK) constitutive law, suitable for large displacements and small deformations, defined by:

$$
S\left(\boldsymbol{u}_{\mathrm{s}}\right)=\mathcal{D}: \boldsymbol{E}\left(\boldsymbol{u}_{\mathrm{s}}\right)
$$

\section{Here, $\mathcal{D}$ is the fourth order tensor of elasticity.}

\subsection{Virtual works with follower forces and SVK constitutive equation}

The problem consists in finding $u_{\mathrm{s}} \in \mathcal{C}_{u}$, with $\mathcal{C}_{u}$ is a subspace of kinematic admissible

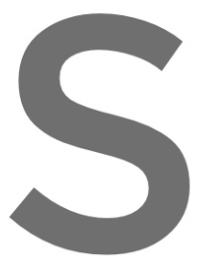
smooth functions, sur
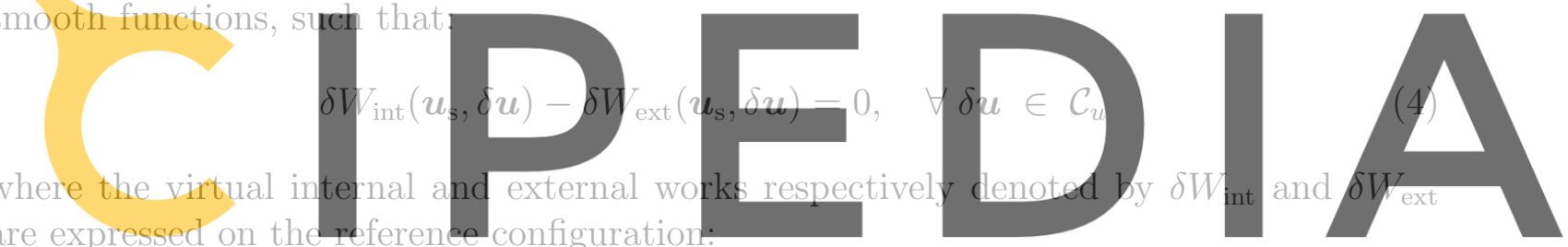

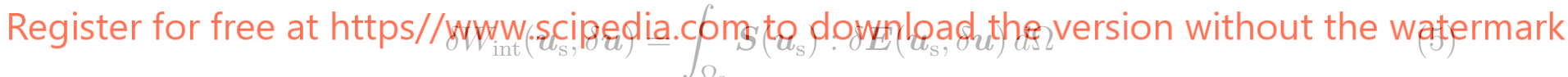

$$
\delta W_{\mathrm{ext}}\left(\boldsymbol{u}_{\mathrm{s}}, \delta \boldsymbol{u}\right)=\int_{\Sigma}-p_{\mathrm{h}}\left(\boldsymbol{u}_{\mathrm{s}}\right) J\left(\boldsymbol{u}_{\mathrm{s}}\right) \boldsymbol{F}^{-\mathrm{T}}\left(\boldsymbol{u}_{\mathrm{s}}\right) \boldsymbol{n} \cdot \delta \boldsymbol{u} d S
$$

where $\Sigma$ is the fluid structure interface given on the reference configuration. On the above equation $\boldsymbol{F}\left(\boldsymbol{u}_{\mathrm{s}}\right)=\boldsymbol{I}+\operatorname{Grad} \boldsymbol{u}_{\mathrm{s}}$ is for the deformation gradient, $J\left(\boldsymbol{u}_{\mathrm{s}}\right)=\operatorname{det} \boldsymbol{F}\left(\boldsymbol{u}_{\mathrm{s}}\right)$ is the Jacobian and $\boldsymbol{n}$ is the unit external normal on the reference configuration. Due to the follower forces, the virtual external work depends on the static solution $\boldsymbol{u}_{\mathrm{s}}$.

\section{Structural dynamic fluctuations around a known prestressed state}

\subsection{Assumption of small fluctuations}

We consider the solution $\boldsymbol{u}_{\text {tot }}$ as a sum of a static known static solution $\boldsymbol{u}_{\mathrm{s}}$ and a dynamic solution $\boldsymbol{u}_{\mathrm{d}}$. The dynamic solution is supposed to be small compared to a characteristic length of the structure $l_{c}$, for example the thickness of the tank (i.e. $\left\|\boldsymbol{u}_{\mathrm{d}}\right\| / l_{c}<<1$ ). 
The total pressure $p_{\text {tot }}$ is also decomposed as a known hydrostatic pressure $p_{\mathrm{h}}$ and a fluctuation of pressure $p_{\mathrm{d}}$ :

$$
\begin{aligned}
& \boldsymbol{u}_{\text {tot }}(H, t)=\boldsymbol{u}_{\mathrm{s}}(H)+\boldsymbol{u}_{\mathrm{d}}(t) \\
& p_{\text {tot }}(H, t)=p_{\mathrm{h}}\left(\boldsymbol{u}_{\mathrm{s}}, H\right)+p_{\mathrm{d}}(t)
\end{aligned}
$$

\subsection{Virtual work principle}

The problem consists in finding the dynamic displacement and pressure $\left(\boldsymbol{u}_{\mathrm{d}}, p_{\mathrm{d}}\right) \in$ $\mathcal{C}_{u} \times \mathcal{C}_{p}$ such that:

$$
\delta W_{\text {acc }}\left(\boldsymbol{u}_{\text {tot }}, \delta \boldsymbol{u}\right)+\delta W_{\text {int }}\left(\boldsymbol{u}_{\text {tot }}, \delta \boldsymbol{u}\right)-\delta W_{\text {ext }}\left(\boldsymbol{u}_{\text {tot }}, \delta \boldsymbol{u}\right)=0, \quad \forall \delta \boldsymbol{u} \in \mathcal{C}_{u}
$$

where the virtual inertia, internal and external works respectively denoted by $\delta W_{\text {acc }}, \delta W_{\text {int }}$ and $\delta W_{\text {ext }}$ are expressed as:

$$
\begin{aligned}
& \delta W_{\text {acc }}\left(u_{\text {tot }}, \delta u\right)=\int_{\Omega_{\mathrm{s}}} \rho_{\mathrm{s}} \ddot{u}_{\text {tot }} \cdot \delta u d \Omega \\
& \delta W_{\text {int }}\left(\boldsymbol{u}_{\text {tot }}, \delta \boldsymbol{u}\right)=\int_{\Omega_{\mathrm{s}}} \boldsymbol{S}\left(\boldsymbol{u}_{\mathrm{tot}}\right): \delta \boldsymbol{E}\left(\boldsymbol{u}_{\mathrm{tot}}, \delta \boldsymbol{u}\right) d \Omega \\
& \delta W_{\text {ext }}\left(\boldsymbol{u}_{\text {tot }}, \delta \boldsymbol{u}\right)=\int_{\Sigma}-p_{\mathrm{h}}\left(\boldsymbol{u}_{\mathrm{s}}\right) J\left(\boldsymbol{u}_{\mathrm{S}}\right) \boldsymbol{F}^{-\mathrm{T}}\left(\boldsymbol{u}_{\mathrm{s}}\right) \boldsymbol{n} \cdot \delta \boldsymbol{u} d S
\end{aligned}
$$
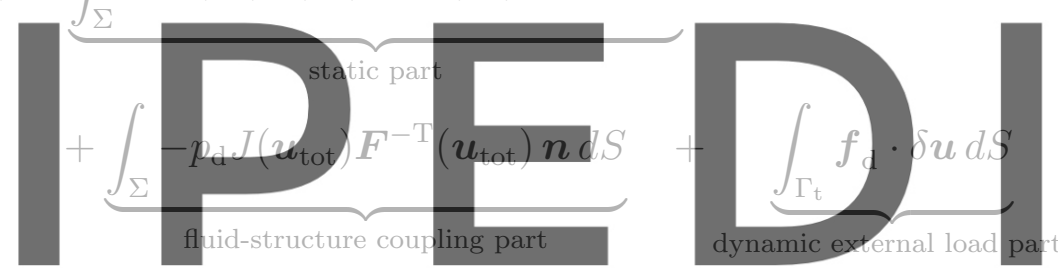

where $\delta u$ represents only an element of the regular space of admissible function. In the

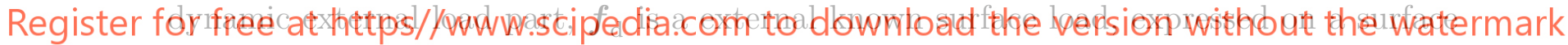

$\Gamma_{t}$, responsible for the dynamic fluctuation of the system. The external work is separated

into a static part, obtained under the assumption of a plane free-surface of the liquid, and two dynamic parts.

\subsection{Linearized structural dynamic problem around a prestressed state}

The linearized problem consists in expressing the virtual work principle considering an assumption on a small variation of displacement around a known static nonlinear state. All the terms in the virtual work principle Eq. (9) are expressed considering a truncation as follow:

$$
\begin{aligned}
& \delta W_{\text {acc }}\left(\boldsymbol{u}_{\text {tot }}, \delta \boldsymbol{u}\right)=0+m\left(\ddot{\boldsymbol{u}}_{\mathrm{d}}, \delta \boldsymbol{u}\right) \\
& \delta W_{\text {int }}\left(\boldsymbol{u}_{\text {tot }}, \delta \boldsymbol{u}\right) \simeq \delta W_{\text {int }}\left(\boldsymbol{u}_{\mathrm{s}}, \delta \boldsymbol{u}\right)+k_{\mathrm{m}}\left(\boldsymbol{u}_{\mathrm{s}} ; \boldsymbol{u}_{\mathrm{d}}, \delta \boldsymbol{u}\right)+k_{\mathrm{g}}\left(\boldsymbol{u}_{\mathrm{s}} ; \boldsymbol{u}_{\mathrm{d}}, \delta \boldsymbol{u}\right) \\
& \delta W_{\text {ext }}\left(\boldsymbol{u}_{\text {tot }}, \delta \boldsymbol{u}\right) \simeq \delta W_{\text {ext }}\left(\boldsymbol{u}_{\mathrm{s}}, \delta \boldsymbol{u}\right)+c\left(\boldsymbol{u}_{\mathrm{s}} ; p_{\mathrm{d}}, \delta \boldsymbol{u}\right)+f(\delta \boldsymbol{u})
\end{aligned}
$$


the semi column is a notation to separated what is known (left) from the linear solution and the associated virtual fields. In Eq. (14), $k_{\mathrm{g}}$ and $k_{\mathrm{m}}$ are respectively the geometrical and material stiffnesses (not detailed here for sake of brevity). In Eqs. (13) and (15), the inertia is denoted by $m$, the coupling $c$ and the linear external load $f$ are given by:

$$
\begin{aligned}
m\left(\ddot{\boldsymbol{u}}_{\mathrm{d}}, \delta \boldsymbol{u}\right) & =\int_{\Omega_{\mathrm{s}}} \rho_{\mathrm{s}} \ddot{\boldsymbol{u}}_{\mathrm{d}} \cdot \delta \boldsymbol{u} d \Omega \\
c\left(\boldsymbol{u}_{\mathrm{s}} ; p_{\mathrm{d}}, \delta \boldsymbol{u}\right) & =\int_{\Sigma}-p_{\mathrm{d}} J\left(\boldsymbol{u}_{\mathrm{s}}\right) \boldsymbol{F}^{-\mathrm{T}}\left(\boldsymbol{u}_{\mathrm{s}}\right) \mathbf{n} \cdot \delta \boldsymbol{u} d S \\
f(\delta \boldsymbol{u}) & =\int_{\Gamma_{\mathrm{t}}} \boldsymbol{f}_{\mathrm{d}} \cdot \delta \boldsymbol{u} d S
\end{aligned}
$$

All terms with an index "d" are associated to the linearized dynamical problem. The dynamical problem consists in finding $\left(\boldsymbol{u}_{\mathrm{d}}, p_{\mathrm{d}}\right) \in \mathcal{C}_{u} \times \mathcal{C}_{p}$, knowing the static nonlinear solution $\boldsymbol{u}_{\mathrm{s}}$, such that:

$$
\underbrace{k_{\tan }\left(\boldsymbol{u}_{\mathrm{s}} ; \boldsymbol{u}_{\mathrm{d}}, \delta \boldsymbol{u}\right)}_{\text {stiffness }}+\underbrace{m\left(\ddot{u}_{\mathrm{d}}, \delta \boldsymbol{u}\right)}_{\text {inertia }}+\underbrace{c\left(\boldsymbol{u}_{\mathrm{s}} ; p_{\mathrm{d}}, \delta \boldsymbol{u}\right)}_{\text {coupling }}=\underbrace{f(\delta \boldsymbol{u})}_{\text {external load }}, \forall \delta \boldsymbol{u} \in \boldsymbol{C}_{\boldsymbol{u}}
$$
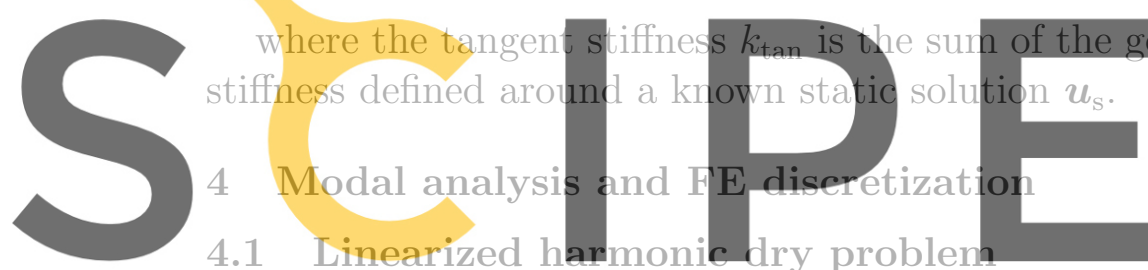

4.1 Linearized harmonic dry problen

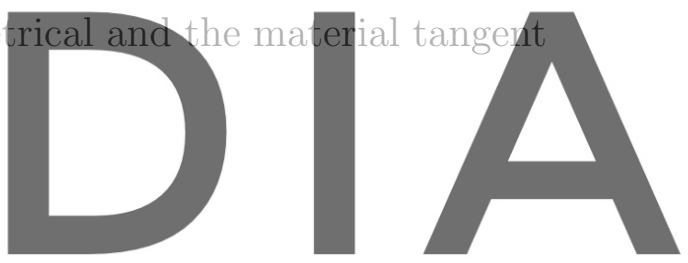

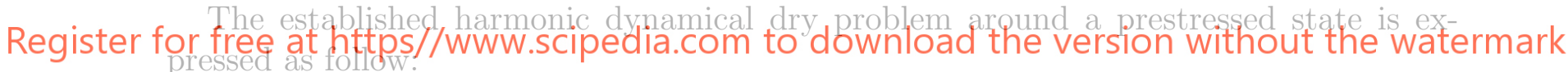

$$
\kappa_{\tan }\left(\boldsymbol{u}_{\mathrm{s}} ; \boldsymbol{u}_{\mathrm{d}}, \delta \boldsymbol{u}\right)-\omega^{2} m\left(\ddot{\boldsymbol{u}}_{\mathrm{d}}, \delta \boldsymbol{u}\right)=f(\delta \boldsymbol{u}), \quad \forall \delta \boldsymbol{u} \in \mathcal{C}_{u}
$$

The finite element discretization leads to the following matrix problem:

$$
\left[\mathbf{K}_{\tan }-\omega^{2} \mathbf{M}\right] \mathbf{u}_{\mathrm{d}}=\mathbf{f}
$$

where $\mathbf{K}_{\tan }$ is the tangent stiffness matrix, $\mathbf{M}$ is the structural mass matrix, $\mathbf{f}_{\mathrm{d}}$ is an external load vector and $\mathbf{u}_{\mathrm{d}}$ is the nodal fluctuation of displacement unknown vector. The following eigenvalue problem is then considered:

$$
\left[\mathbf{K}_{\tan }-\omega^{2} \mathbf{M}\right] \mathbf{u}=\mathbf{0}
$$

In the following, the matrices properties are supposed to be verified to obtain eigenvectors and eigenvalues $\left\{\mathbf{u}_{\alpha}, \omega_{\alpha}^{2}\right\}_{\alpha=1 \ldots m}$ from Eq. (22) where $\omega_{\alpha}$ are positives. In the following the Greek-letter indices correspond to the reduced coordinates. An approximation of the 
displacement fluctuation $\mathbf{u}_{\mathrm{d}}$ is expressed as a linear combinations of the $m$ first eigenmodes such that:

$$
\mathbf{u}_{\mathrm{d}} \simeq \sum_{\alpha=1}^{n_{\mathrm{d}}} q_{\alpha} \mathbf{u}_{\alpha}=\mathbf{\Psi q} \quad \text { with } \quad \boldsymbol{\Psi}=\left[\begin{array}{ccc}
\vdots & & \vdots \\
\mathbf{u}_{1} & \ldots & \mathbf{u}_{m} \\
\vdots & & \vdots
\end{array}\right]_{n_{\mathrm{s}} \times m}
$$

where $n_{\mathrm{s}}$ is the number of degrees of freedom on the structure, the $q_{\alpha}$ are the generalized coordinates and $\mathbf{q}$ the associated unknown vector. $\boldsymbol{\Psi}$ is the matrix containing the prestressed modal basis, with a mass normalization such that $\mathbf{u}_{\alpha}^{\mathrm{T}} \mathbf{M} \mathbf{u}_{\alpha}=1$. The projection of the linearized problem Eq. (20) leads to the following reduced problem:

$$
\left[\mathbb{K}_{\mathrm{r}}-\omega^{2} \mathbf{M}_{\mathrm{r}}\right] \mathrm{q}=\mathfrak{f}_{\mathrm{r}}
$$

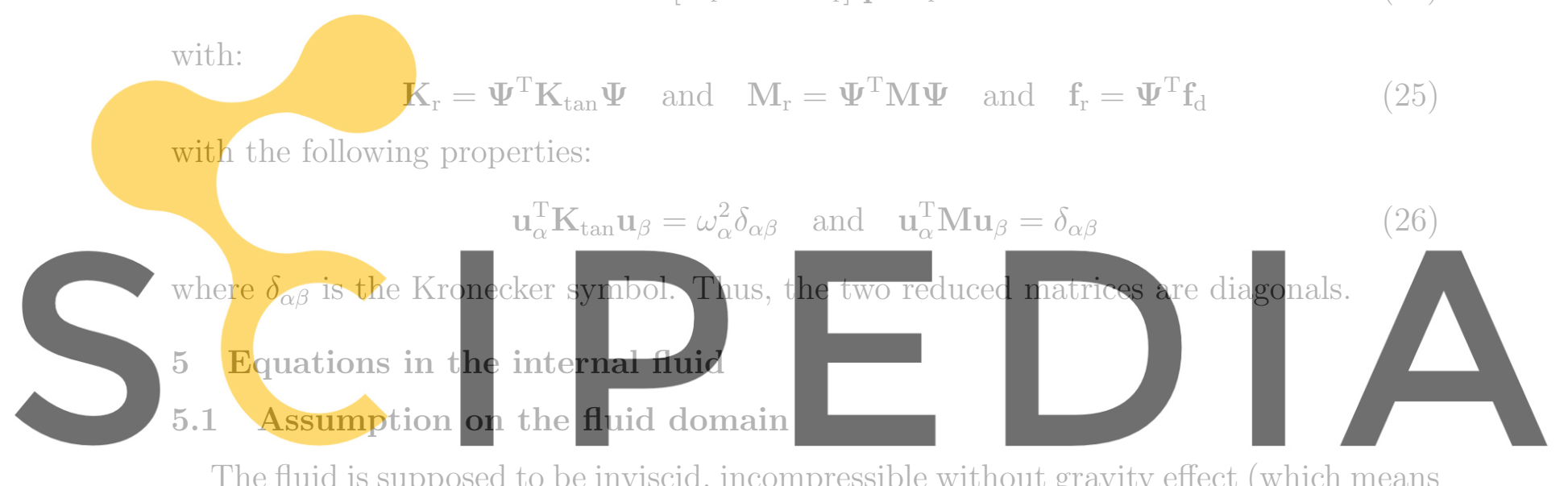

The fluid is supposed to be inviscid, incompressible without gravity effect (which means

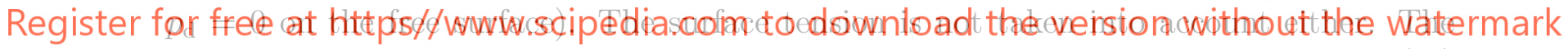
current fluid domain $\Omega_{\mathrm{f}}^{\text {cur }}$ depend on the fluid height $H$ and the static displacement $u(H)$ at the fluid structure interface $\Sigma$ on a current configuration. For sake of convenience, the reference fluid-structure interface does not change in function of the fluid height. In addition, the displacement of the fluid-structure interface is supposed to be small so the effect of $\boldsymbol{u}_{\mathrm{s}}$ on the fluid domain is neglected. In the following, only the fluid height is supposed to have an effect on the fluid domain such that:

$$
\Omega_{\mathrm{f}}^{\text {cur }}(H) \simeq \Omega_{\mathrm{f}}^{\text {ref }}(H) \equiv \Omega_{\mathrm{f}}
$$

where $\Omega_{\mathbf{f}}^{\text {ref }}(H)$ is one reference configuration of the fluid domain.

\subsection{Harmonic fluctuation of pressure as a finite sum of induced potentials}

The fluctuation of pressure problem on the fluid consists in finding $\left(p_{\mathrm{d}}, \boldsymbol{u}_{\mathrm{d}}\right) \in \mathcal{C}_{p} \times \mathcal{C}_{u}$ such that :

$$
h\left(p_{\mathrm{d}}, \delta p\right)+\rho_{\mathrm{f}} c\left(\boldsymbol{u}_{\mathrm{s}} ; \delta p, \ddot{\boldsymbol{u}}_{\mathrm{d}}\right)=0, \quad \forall \delta p \in \mathcal{C}_{p}
$$


where $\rho_{\mathrm{f}}$ is the mass dentity of the fluid, $h$ is a bi-linear form such that:

$$
h\left(p_{\mathrm{d}}, \delta p\right)=\int_{\Omega_{\mathrm{f}}} \operatorname{Grad} p_{\mathrm{d}} \cdot \operatorname{Grad} \delta p d \Omega
$$

Then, we consider the dynamic pressure as a linear combination of potential, such that:

$$
p_{\mathrm{d}} \simeq-\rho_{\mathrm{f}} \omega^{2} \sum_{\alpha=1}^{n_{\mathrm{d}}} q_{\alpha} \varphi_{\alpha}
$$

where the potential $\varphi_{\alpha}$ corresponds to the potential of displacement to a given eigenmode $\boldsymbol{u}_{\alpha}$ regarding the following set of equations:

$$
\begin{aligned}
\Delta \varphi_{\alpha} & =0 & & \text { in } \Omega_{\mathrm{f}} \\
\operatorname{Grad} \varphi_{\alpha} \cdot J\left(u_{\mathrm{s}}\right) \mathbb{F}^{-\mathrm{T}}\left(u_{\mathrm{s}}\right) n & =u_{\alpha} \cdot J\left(u_{\mathrm{s}}\right) \mathbb{F}^{-\mathrm{T}}\left(u_{\mathrm{s}}\right) n & & \text { on } \Sigma \\
\varphi_{\alpha} & =0 & & \text { on } \Gamma
\end{aligned}
$$

Considering a variational formulation and a finite element discretization, the computation of the potential of displacement are obtain considering the following linear system of

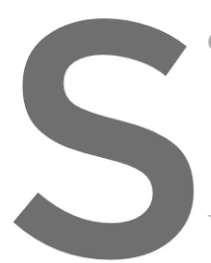
equation:
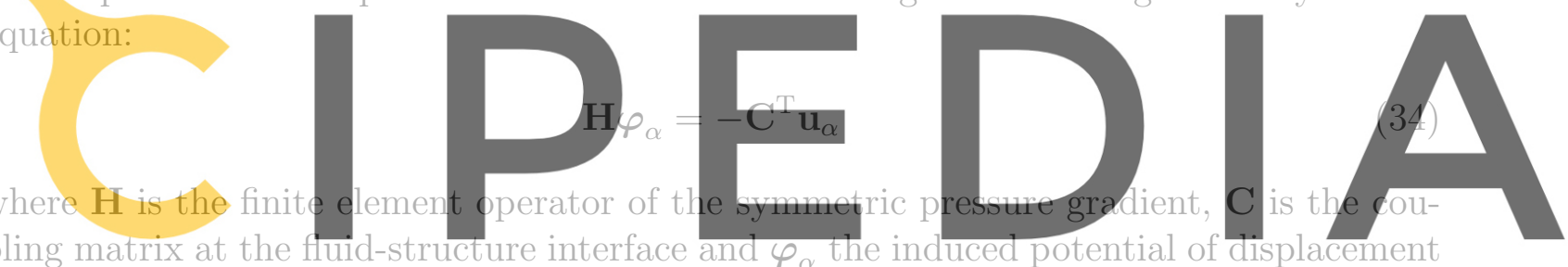

Register for free at hittps//www.scipedia.com to download the version without the watermark

6 Reduced hydroelastic eigenvalue problem with prestressing

Considering the prestressed based $\boldsymbol{\Psi}$ and the associated fluid responses concatenated in a matrix $\boldsymbol{\Phi}$, one can express the following reduced eigenproblem from the coupling term Eq. (22):

$$
\left[\mathbf{K}_{\mathrm{r}}-\omega^{2}\left(\mathbf{M}_{\mathrm{r}}+\mathbf{M}_{\mathrm{ra}}\right)\right] \mathbf{q}=\mathbf{0}
$$

where the reduced added mass matrix is given by the following expression:

$$
\mathbf{M}_{\mathrm{ra}}=-\rho_{\mathrm{f}} \boldsymbol{\Psi}^{\mathrm{T}} \mathbf{C} \boldsymbol{\Phi} \text { with } \boldsymbol{\Phi}=\left[\begin{array}{ccc}
\vdots & & \vdots \\
\boldsymbol{\varphi}_{1} & \ldots & \boldsymbol{\varphi}_{m} \\
\vdots & & \vdots
\end{array}\right]_{n_{\mathrm{f}} \times m}
$$

where $n_{\mathrm{f}}$ is the number of degrees of freedom in the fluid. The reduced eigenvalue problem (35) leads to the computation of the reduced eigenvectors and the hydroelastic eigenvalues 
$\left\{\mathbf{q}_{\gamma}, \omega_{\gamma}^{2}\right\}_{\gamma=1 \ldots n_{\mathrm{d}}}$ where $\omega_{\gamma}$ are the hydroelastic pulsation of the prestressed problem. The advantage of this approach relies on the computation of the reduced added mass matrix $\mathbf{M}_{\text {ra }}$ which depends on $\boldsymbol{\Phi}$ such that:

$$
\Phi=\mathbf{H}^{-1} \mathbf{C}^{\mathrm{T}} \boldsymbol{\Psi}
$$

No explicit inverse of the operator $\mathbf{H}$ is computed (with respect to the boundary condition on the free surface) considering the $m$ linear systems of equations from Eq. (34). It can be highlighted that $\mathrm{M}_{\mathrm{ra}}$ is a symmetric matrix, but not diagonal.

\section{Example : circular plate under a column of water}

The numerical problem presented in this section is based on an experimental study [4]. The problem consists in evaluating the prestressed hydroelastic pulsations of a clamped circular plate under a column of incompressible liquid (see Fig 2). It is a problem parameterized by the fluid height $H$. All the numerical results are generated with the FEniCS package [3].
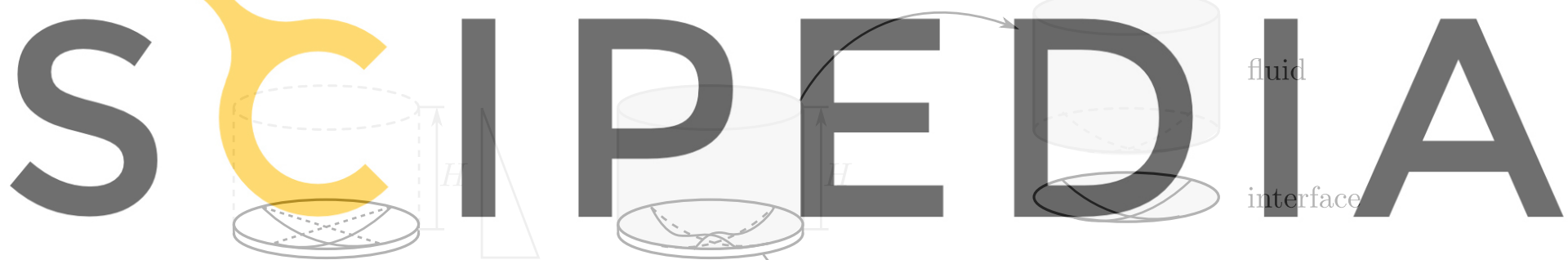

Register for free at https//www.scipedia.com to download the version-witholit the watermark

(a)

(b)

Figure 2: Prestressed vibrations of a circular plate under a column of liquid. (a) Nonlinear static analysis considering a hydrostatic follower force and (b) linearized hydroelastic vibrations around prestressed state. For each fluid height increment, a new fluid mesh, coincident with the solid mesh at the interface is generated. 


\subsection{Nonlinear static analysis with hydrostatic follower forces}

The nonlinear static problem is illustrated in Fig. 3. A three dimensional circular clamped plate is subject to a hydrostatic follower forces driven by the fluid height.
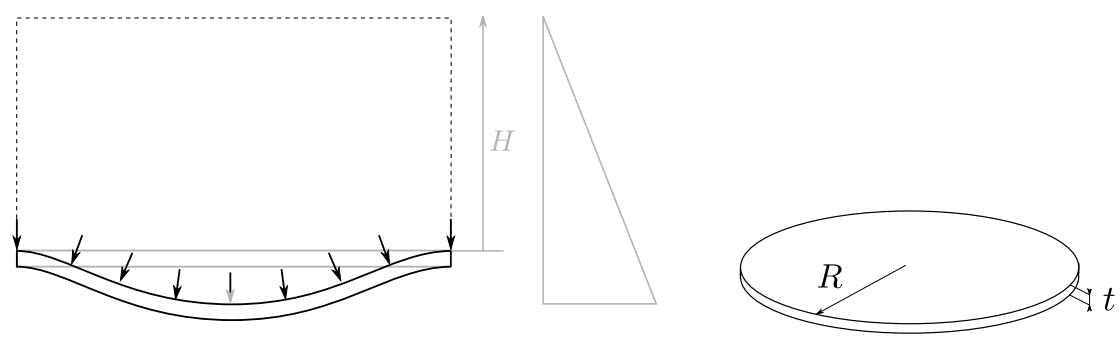

Figure 3: Definition of the nonlinear static problem with an hydrostatic follower forces. The fluid pressure is defined as $p_{\mathrm{h}}(H)=-\rho g(z-H)$ with $\rho_{\mathrm{f}}=1 \times 10^{3} \mathrm{~kg} / \mathrm{m}^{-3}, g=9.81$ $\mathrm{m} . \mathrm{s}^{-2}$ and $H \in[0,250] \mathrm{mm}$. The plate geometrical parameters are $R=0.144 \mathrm{~m}$ and $t=0.35 \mathrm{~mm}$. The material parameters are the Young modulus $E=6.9 \times 10^{7}$, the Poisson ratio $\nu=0.38$ and the mass density $\rho_{\mathrm{s}}=1.4 \times 10^{3} \mathrm{~kg} / \mathrm{m}^{-3}$.
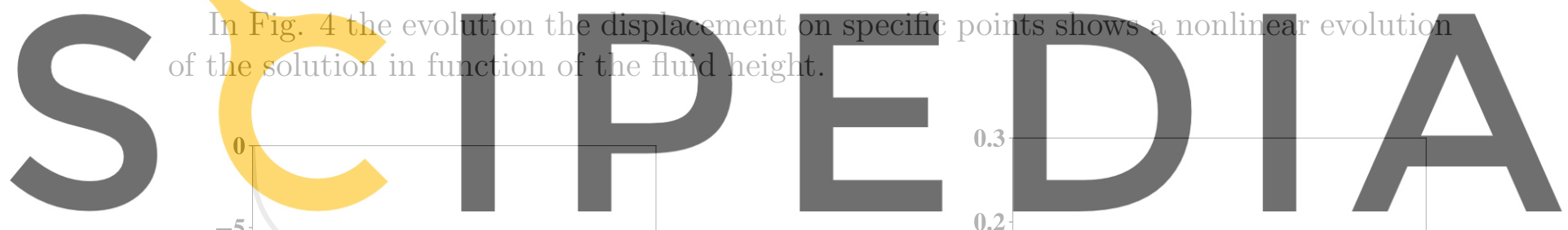

Register fờ free at https//www.scipedia.com to download̆ the version without the watermark
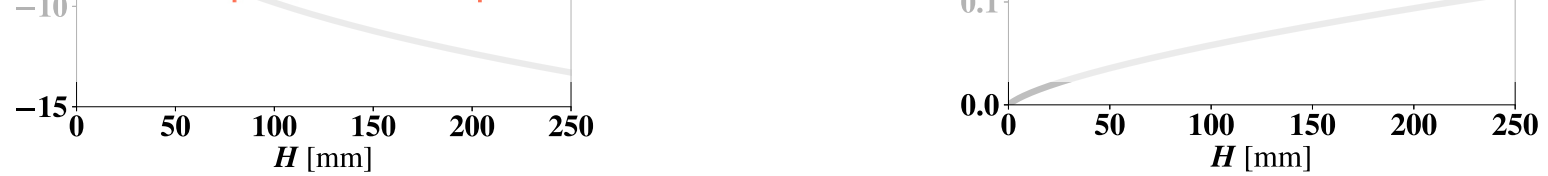

Figure 4: Evolution of transversal displacement $u_{z}$ (left) at the center of the plate $\boldsymbol{x}_{c}=$ $(0.0,0.0,0.0)$ and evolution of the radial displacement $u_{x}$ (right) at $\boldsymbol{x}_{r}=(R / 2,0.0,0.0)$, considering 200 uniformed fluid height steps with $\Delta h=1.25 \mathrm{~mm}$.

Those results have been obtained considering a structural mesh with three dimensional quadratic tetrahedron. 


\subsection{Reduced prestressed hydroelastic modal analysis and fluid response}

Knowing the nonlinear static solution, the hydroelastic modes are computed as illustrated in Fig. 5. A fluid mesh have been generated for each known structural configurations.

Nonlinear static analysis

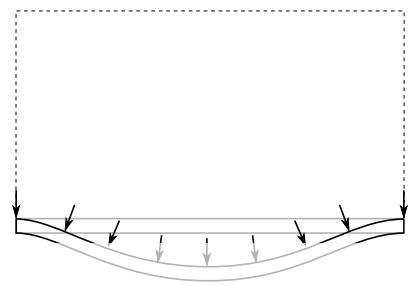

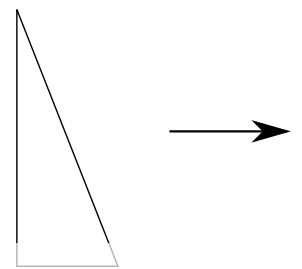

\section{Linearized hydroelasticity}

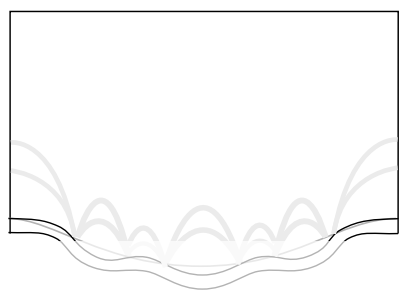

Figure 5: Methodology in two steps : (i) nonlinear static analysis and (ii) linearized hydroclastic vibrations around the prestressed state.

At each fluid height, a modal prestressed structural basis is generated. In Fig. 6, the

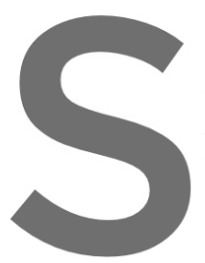

linear modal basis wi

each fluid heigh step

presented for sake of
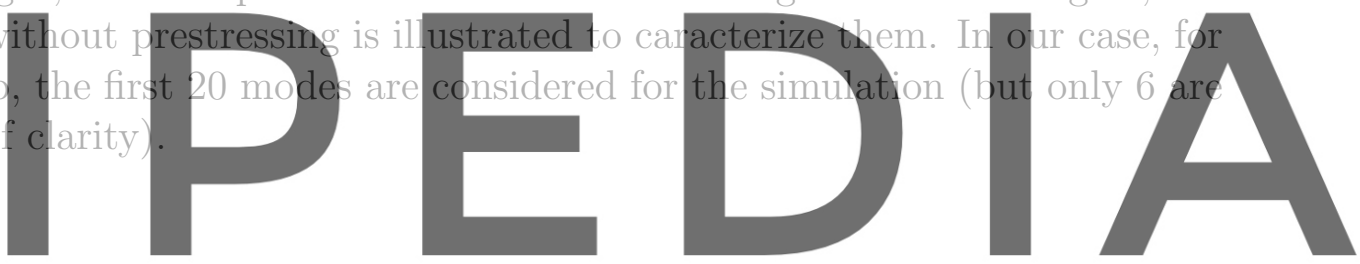

Register for free at https//www.scipedia.com to download the version without the watermark

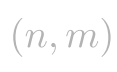

Symbols
$(0,1)$

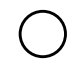

$(1,1)$

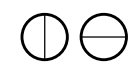

$(2,1)$

$(0,2)$

(2)

Figure 6: Mode characterization in terms of circumferential waves $n$ and radial waves $m$ and associated symbols for $H=0$.

Knowing the structural prestressed basis, the potential of displacements associated with each structural modes are computed with a linear system of equation. In Fig. 7 an example of fluid response is given for various liquid height. The reduced added mass matrix is finally expressed knowing the prestressed structural basis and the associated potential of displacement fluid responses. 


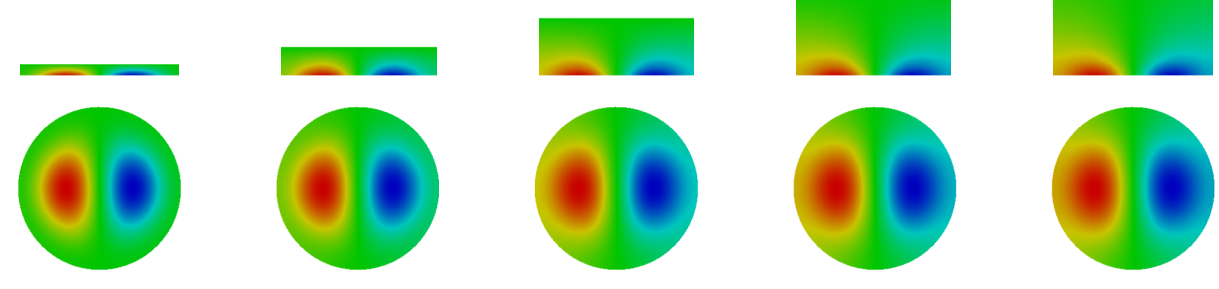

Figure 7: Fluid potential of displacement $\varphi_{1}(H)$ obtained as a response of the prestressed structural mode mode $(0,1)$ for various fluid height.

\subsection{Numerical analysis : three numerical test cases}

Three test cases are analyzed here. The first one consists in evaluating only the prestressed effect on the eigenfrequencies of the plate (see Fig. 8 (a)). The second one consists in evaluating only the effect of the fluctuated fluid without prestressing (see Fig. 8 (b)). The last case take into account both effects (see in Fig. 8 (c)) and show very good agreement with the experimental results given in [4].

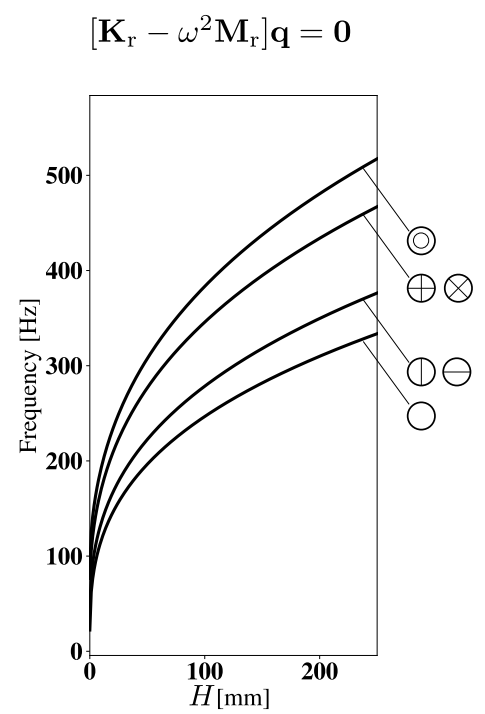

(a)

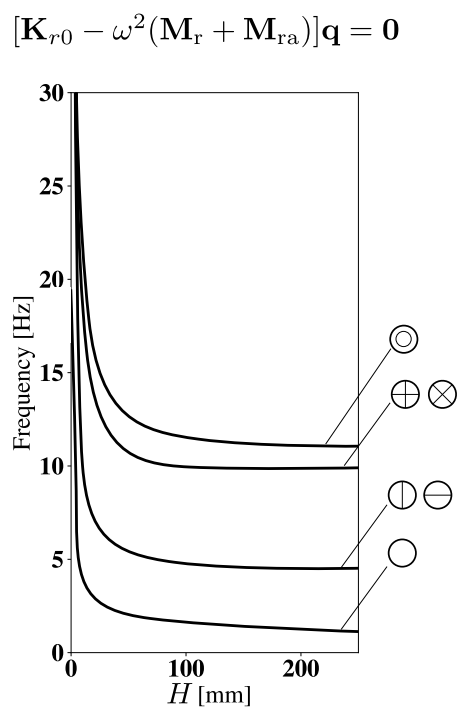

(b)

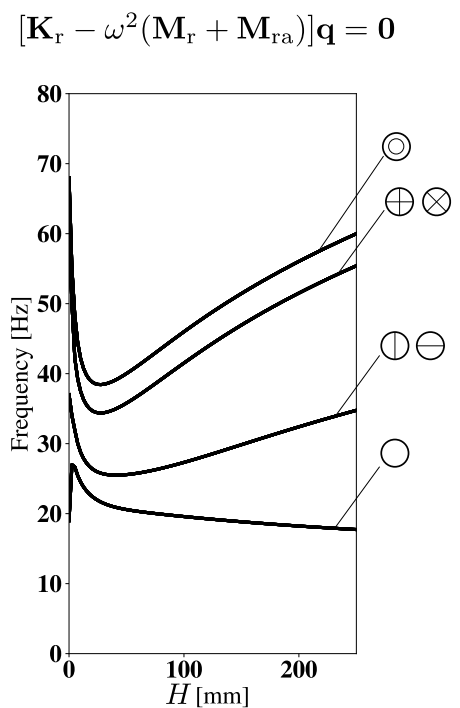

(c)

Figure 8: Evolution of 6 eigenfrequencies for varying fluid height : (a) linearized prestressed analysis only; (b) added mass effect only (c) prestressing and added mass effect. 
It is worth to mentioned that only the third case results are in agreement with the results observed experimentally. The first two cases are manufactured simulations which allow to isolate physical effects from the model. In this specific configuration, the curve from Fig. 8 (a) show an increasing evolution of the given resonance frequencies in function of the fluid height. For this geometry, the pressure acting on the plate have a stiffening effect on system. On the other hand, the curve on Fig. 8 (b) shows a decrease of the hydroelastic frequencies in function of the fluid height. Indeed, it is due to the influence of the kinetic energy of the fluid displaced on the structural dynamic behavior. It is observed that for the modes $(1,1)$ and $(2,1)$, the eigenfrequencies curves reach a plateau in function of $H$. For those modes, it seems that the fluid potential of displacement fluctuations remains near the fluid structure interface. Finally, the last case shows a decreasing and then an increasing evolution of the natural hydroelastic frequencies (except for the mode $(0,0)$ which have an opposite behavior). This is in very good agreement with the the observations from the literature which validate the model developed for the computation of prestressed hydroelastic vibrations.

\section{Conclusion and perspectives}

In this paper, a projection-based reduced order model for prestressed hydroelastic vibrations have been presented. First the linearized dynamic fluid-structure interaction problem around a prestressed state have been developed. Then, a projection of the problem on an associated prestressed modal basis lead to the expression of the problem in terms of generalized coordinates. The computation of a reduced tangent and added mass matrix allow a fast computation of the firt eigenfrequencies of the prestressed hydroelastic depending of the number of prestressed dry modes considered. A balance between the frequency range of interest and the number prestressed modes selected have to be addressed. A numerical example shows very good agreements in comparison with Chiba experimental studies [4]. Further investigations to construct a tangent stiffness matrix as a finite sum of reduced operators are expected in the future.

\section{REFERENCES}

[1] Morand, H. J.-P. and Ohayon, R. Fluid-Structure Interaction. Wiley, (1995).

[2] Hoareau, C. and Deü, J.-F., Nonlinear equilibrium of partially liquid-filled tanks: A finite element/level-set method to handle hydrostatic follower forces. Int. J. Non Linear Mech. (2019) 113:112-127.

[3] Alnaes, M. S. et al., The FEniCS Project Version 1.5. Arch. Num. Soft. (2015) 3.

[4] Chiba, M., Nonlinear hydroelastic vibration of a cylindrical tank with an elastic bottom, containing liquid. part i : Experiment. J. Fluids Struct. (1992) 6:181-206. 